\title{
Validity of the relationship between mobility group box-1 protein and poor post-cardiac arrest neurological outcome during renal replacement therapy
}

\author{
Patrick M. Honore ${ }^{1 *}$, David De Bels ${ }^{1}$, Luc Kugener ${ }^{1}$, Sebastien Redant ${ }^{1}$, Rachid Attou ${ }^{1}$, Andrea Gallerani ${ }^{1}$ and \\ Herbert D. Spapen ${ }^{2}$
}

See related research by Sugita et al., https://ccforum.biomedcentral.com/articles/10.1186/s13054-017-1828-5.

Recently in Critical Care, Sugita et al. confirmed the previously reported positive association between high HMGB-1 (high-mobility group box-1) levels and a poor neurological outcome in post-cardiac arrest (post-CA) patients [1]. Although we applaud this excellent study, we would like to make some comments. Brain damage after out-of-hospital CA is a leading cause of mortality and long-term neurological disability in survivors. Primary injury is caused by an immediate shutdown of cerebral blood flow. In the hours and days following CA, systemic ischemia/reperfusion (I/R)-induced processes may culminate in secondary neuronal cell death [2]. HMGB-1, a typical damage-associated molecular pattern protein, is crucial in inflammation and is critically involved in thrombosis and also mediates systemic organ I/R. Interestingly, almost half of the patients resuscitated after CA may develop acute kidney injury (AKI). More than 75\% of AKI episodes occurred within 3 days after $\mathrm{CA}$ and about one third of these patients eventually needed continuous renal replacement therapy (CRRT) [3]. Sugita et al. did not mention the incidence of
AKI or need of (C)RRT. However, given the high number of patients in shock (75\% of patients with a GlasgowPittsburgh Cerebral Performance Categories Scale (CPC) score of 3 to 5 were on vasopressors!), it is reasonable to assume that a substantial number developed AKI or needed (C)RRT or both. With a molecular weight of $30 \mathrm{kDa}$, HMGB-1 is not eliminated by convective CRRT. However, Yumoto et al. showed that more than 90\% of HMGB-1 was cleared from the circulation by highly adsorbent surfacetreated polyacrylonitrile and polymethylmethacrylate membranes but that no such removal was observed with the less adsorptive polysulfone membranes [4]. Although polysulfone hemofilters are most commonly used for CRRT in Japan [5], implementing highly adsorbent filters would result in much lower serum HMGB-1 levels in post-CA patients. This not only would challenge the conclusions made by Sugita et al. but also could affect patient outcome since an extensive elimination of HMGB-1 may theoretically subdue the I/R-related post-CA injurious process.

\section{Authors' response}

Atsushi Sakurai, Kosaku Kinoshita, Atsunori Sugita, Nobutaka Chiba, Junko Yamaguchi, Tsukasa Kuwana, Nami Sawada and Satoshi Hori

Following the revision of our data, we identified 12 of 128 patients to be treated with continuous renal replacement therapy (CRRT), including 7 who had been on haemodialysis therapy prior to the cardiac arrest (CA).

\footnotetext{
* Correspondence: patrick.honore@chu-brugmann.be

${ }^{1}$ ICU Department, Centre Hospitalier Universitaire Brugmann-Brugmann

University Hospital, Place Arthur Van Gehucthen, 41020 Brussels, Belgium

Full list of author information is available at the end of the article
}

Only 5 patients (3.9\%) were treated with CRRT for renal failure in our study.

Whether elimination of HMGB1 could contribute to the amelioration of the neurological deficits in patients with post-cardiac arrest syndrome (PCAS) is a crucial question. HMGB1 clearance from the brain may afford brain protection in PCAS patients. Considering the small molecular weight of HMGB1 and that CA induces 
the blood-brain barrier breakdown in the early phase of post-resuscitation, it is possible that serum HMGB1 may invade the brain through the broken $\mathrm{BBB}$ and promote the inflammatory pathophysiology of the ischemic reperfusion brain injury. $\mathrm{Xu}$ et al. reported that the neurological deficits significantly improved in rats resuscitated from CA following the injection of the anti-HMGB1 antibody into their cerebral ventricles [1]. Another study reported that the intravenous injection of an anti-HMGB1 antibody improved the cerebral haemorrhage-induced neurological deficits in rats [2]. Therefore, these findings suggest that the systemic clearance of HMGB1 might also ameliorate post-cardiac arrest brain injury.

As Dr. Honore proposed, using the CRRT for clearance of serum HMGB1 may be a promising candidate method for the treatment of PCAS patients. During the process of brain injury, damage-associated molecular patterns, including HMGB1, are released from the injured neurons and activate inflammation, which results in further neural and glial death during stroke [3]. In the present study, the peaks of serum HMGB1, interleukin- 6 and neuron-specific enolase were 0,24 and $48 \mathrm{~h}$ from resuscitation, respectively [4], indicating that a pathologic mechanism similar to stroke may occur in PCAS patients. In contrast HMGB1 was higher in the cerebrospinal fluid of PCAS patients than in their serum at $48 \mathrm{~h}$ after resuscitation [5]. These results suggest that, in addition to the primary inflammatory brain injury, HMGB1 may also promote the secondary inflammatory brain injury in PCAS patients. However, when the primary brain damage is severe, decreasing HMGB1 activity in PCAS patients will not be effective. Therefore, both the timing of the treatment and the severity of brain injury should be taken into consideration when investigating HMGB1 clearance treatment therapy for PCAS patients.

\section{Abbreviations}

AKl: Acute kidney injury; CA: Cardiac arrest; (C)RRT: (Continuous) renal replacement therapy; HMGB-1: High-mobility group box-1; I/R: Ischemia/ Reperfusion

\section{Acknowledgments}

None.

\section{Funding}

None.

Availability of data and materials

Not applicable.

Authors' contributions

$\mathrm{PMH}$ and HDS designed the paper. All authors participated in drafting and reviewing. All authors read and approved the final version of the manuscript.
Consent for publication

Not applicable.

Competing interests

The authors declare that they have no competing interests.

\section{Publisher's Note}

Springer Nature remains neutral with regard to jurisdictional claims in published maps and institutional affiliations.

\section{Author details}

${ }^{1}$ ICU Department, Centre Hospitalier Universitaire Brugmann-Brugmann University Hospital, Place Arthur Van Gehucthen, 41020 Brussels, Belgium.

${ }^{2}$ Ageing \& Pathology Research Group, Vrije Universiteit Brussel, 101

Laarbeeklaan, 1070 Brussels, Belgium.

Received: 20 November 2018 Accepted: 21 December 2018

Published online: 12 March 2019

References

1. Sugita A, Kinoshita K, Sakurai A, Chiba N, Yamaguchi J, Kuwana T, et al. Systemic impact on secondary brain aggravation due to ischemia/reperfusion injury in post-cardiac arrest syndrome: a prospective observational study using high-mobility group box 1 protein. Crit Care. 2017;21:247.

2. Sekhon MS, Ainslie PN, Griesdale DE. Clinical pathophysiology of hypoxic ischemic brain injury after cardiac arrest: a "two-hit" model. Crit Care. 2017:21:90.

3. Tujjar O, Mineo G, Dell'Anna A, Poyatos-Robles B, Donadello K, Scolletta S, et al. Acute kidney injury after cardiac arrest. Crit Care. 2015;19:169.

4. Yumoto M, Nishida O, Moriyama K, Shimomura Y, Nakamura T, Kuriyama N, et al. In vitro evaluation of high mobility group box 1 protein removal with various membranes for continuous hemofiltration. Ther Apher Dial. 2011;15: 385-93

5. Yasuda H, Sekine K, Abe T, Suzaki S, Katsumi A, Harada N, et al. Comparison of two polysulfone membranes for continuous renal replacement therapy for sepsis: a prospective cross-over study. Renal Replacement Therapy. 2018;4:6. 